\title{
Blockade of canonical Wnt signalling ameliorates experimental dermal fibrosis
}

\author{
Christian Beyer, ${ }^{1}$ Helena Reichert, ${ }^{1}$ Hümeyra Akan, ${ }^{1}$ Tatjana Mallano, ${ }^{1}$ \\ Amelie Schramm, ${ }^{1}$ Clara Dees, ${ }^{1}$ Katrin Palumbo-Zerr, ${ }^{1}$ Neng Yu Lin, ${ }^{1}$ Alfiya Distler, \\ Kolja Gelse, ${ }^{2}$ John Varga, ${ }^{3}$ Oliver Distler, ${ }^{4}$ Georg Schett, ${ }^{1}$ Jörg H W Distler ${ }^{1}$
}

\begin{abstract}
- Additional material is published online only. To view please visit the journal online (http://dx.doi.org/10.1136/ annrheumdis-2012-202544).

${ }^{1}$ Department of Internal Medicine 3, Institute for Clinical Immunology, University of Erlangen-Nuremberg, Erlangen, Germany ${ }^{2}$ Department of Orthopaedic Trauma Surgery, University Hospital Erlangen, Erlangen, Germany

${ }^{3}$ Division of Rheumatology, Northwestern University Feinberg School of Medicine, Chicago, Illinois, USA ${ }^{4}$ Department of Rheumatology, University Hospital Zurich, Zurich, Switzerland

\section{Correspondence to Dr Jörg H W Distler, Department of Internal Medicine 3, Institute for Clinical Immunology, University of Erlangen-Nuremberg, Ulmenweg 18, Erlangen 91054, Germany; joerg.distler@uk-erlangen.de}

Accepted 19 March 2013 Published Online First 17 April 2013

\section{ABSTRACT}

Background and objectives Fibrosis is a major socioeconomic burden, but effective antifibrotic therapies are not available in the clinical routine. There is growing evidence for a central role of Wnt signalling in fibrotic diseases such as systemic sclerosis, and we therefore evaluated the translational potential of pharmacological Wnt inhibition in experimental dermal fibrosis. Methods We examined the antifibrotic effects of PKF118-310 and ICG-001, two novel inhibitors of downstream canonical Wnt signalling, in the models of prevention and treatment of bleomycin-induced dermal fibrosis as well as in experimental dermal fibrosis induced by adenoviral overexpression of a constitutively active transforming growth factor (TGF)- $\beta$ receptor I.

Results PKF118-310 and ICG-001 were well tolerated throughout all experiments. Both therapeutic approaches showed antifibrotic effects in preventing and reversing bleomycin-induced dermal fibrosis as measured by skin thickness, hydroxyproline content and myofibroblast counts. PKF118-310 and ICG-001 were effective in inhibiting TGF- $\beta$ receptor I-driven fibrosis as assessed by the same outcome measures.

Conclusions Blockade of canonical Wnt signalling by PKF118-310 and ICG-001 showed antifibrotic effects in different models of skin fibrosis. Both therapies were well tolerated. Although further experimental evidence for efficacy and tolerability is necessary, inhibition of canonical Wnt signalling is a promising treatment approach for fibrosis.

\section{INTRODUCTION}

Fibrosis is characterised by scaring of various tissues, which arises from excess deposition of extracellular matrix components such as collagens. During the course of fibrotic disease, progressive fibrosis disrupts the physiological tissue architecture and causes organ failure. ${ }^{1}$ Despite its major impact on disease burden, therapies that effectively reduce fibrosis are not available in the clinical routine.

Recent work has identified canonical Wnt signalling as one of the central profibrotic signalling pathways in fibrotic diseases, including systemic sclerosis (SSc). ${ }^{2-9}$ SSc is often considered a prototypical fibrotic disease, since it affects the skin and many internal organs. ${ }^{10}{ }^{11}$ In SSc, overexpression of Wnt-1 and Wnt-10b as well as downregulation of the endogenous Wnt inhibitor dickkopf-1 by transforming growth factor (TGF)- $\beta$, lead to the activation of canonical Wnt signalling. ${ }^{2-5}$ On receptor activation, $\beta$-catenin, the central integrator of the canonical Wnt pathway, is stabilised and translocates into the nucleus. Complex formation of $\beta$-catenin with T cell factor (TCF) transcription factors and subsequent recruitment of co-activators (eg, CREB-binding protein (CBP) and p300) initiate the transcription process of Wnt target genes. $^{12}$

Preclinical studies highlight the potent profibrotic effects of canonical Wnt signalling in skin fibrosis. Overexpression of Wnt-10b as well as genetic or pharmacological stabilisation of $\beta$-catenin cause spontaneous and progressive skin fibrosis. ${ }^{2-5}$ By contrast, fibroblast-specific deletion of $\beta$-catenin blocks the development of experimental fibrosis. ${ }^{2}$

Considering the strong profibrotic effects of canonical Wnt signalling and the urgent need for potent antifibrotic therapies, we evaluated pharmacological strategies to inhibit the canonical Wnt cascade in different experimental models of dermal fibrosis.

\section{MATERIAL AND METHODS}

Prevention and treatment of bleomycin-induced dermal fibrosis

Skin fibrosis was induced in 6-week-old DBA/2 mice (Janvier SAS, Le Genest Saint Isle, France) by subcutaneous injections of bleomycin as described previously. $^{2} 31314$ ICG-001 (ENZO Life Sciences, Lörrach, Germany) was diluted in phosphate buffered saline (PBS) and injected intraperitoneally once daily to a final concentration of $4 \mathrm{mg} / \mathrm{kg}$ bodyweight. PKF118-310 (Sigma-Aldrich, Munich, Germany) was diluted in PBS and injected subcutaneously in the field of bleomycin treatment once daily to a final concentration of $2 \mathrm{mg} / \mathrm{kg}$ bodyweight (injection volume $50 \mu \mathrm{l}$ ). The doses were based on previously published dose ranges in investigations of other diseases. ${ }^{15-17}$

Dermal fibrosis induced by attenuated adenoviruses overexpressing a constitutively active TGF- $\beta$ receptor I

Dermal fibrosis was induced in 4-week-old C57BL/ 6 mice (Janvier SAS) by intracutaneous injections of replication-deficient type $\mathrm{V}$ adenoviruses overexpressing a constitutively active TGF- $\beta$ receptor type I (AdTBRIact). The adenoviral vectors $\left(6.7 \times 10^{7}\right.$ infectious units, IFU) were injected at 4 weeks and 8 weeks of age, and mice were 
sacrificed at the age of 12 weeks. ${ }^{3}$ ICG-001 and PKF118-310 were applied according to their application in the bleomycin model.

\section{Analysis of murine skin}

Dermal thickness, $\alpha$-smooth muscle actin and hydroxyproline content were analysed as described previously. ${ }^{2} 31314$ Toxicity was assessed by a semi-quantitative clinical compound score including posture, activity, texture of the fur and skin integrity (see online supplementary table S1).

\section{Statistics}

All data are presented as median with IOR, and differences between the groups were tested by the non-parametric MannWhitney $U$ test. A p value of less than 0.05 was considered statistically significant.

\section{RESULTS}

Interference with canonical Wnt signalling prevents and reverses bleomycin-induced dermal fibrosis

We evaluated the antifibrotic effects of two novel inhibitors of canonical Wnt signalling. While PKF118-310 inhibits the $\beta$-catenin/TCF transcription factor interaction, ICG-001 interferes with the recruitment of co-activators to $\beta$-catenin. We first analysed the effects of the two Wnt inhibitors in the inflammatory model of bleomycin-induced dermal fibrosis. Treatment with PKF118-310 and ICG-001 effectively inhibited canonical Wnt signalling with reduced mRNA levels of the target gene axin-2 (data not shown). Both compounds were well tolerated, and mice showed normal clinical scores (consistently 0 according to online supplementary table S1) and constant weight (see online supplementary figure S1A,B). Targeting Wnt signalling by PKF118-310 or ICG-001 prevented bleomycin-induced dermal fibrosis as assessed by dermal thickness, hydroxyproline content and myofibroblast counts (figure 1A-D). Treatment with the Wnt inhibitors did not prevent atrophy of the subcutaneous fat tissue (see online supplementary figure S2A), and inflammatory infiltrates, $\mathrm{T}$ cell and $\mathrm{B}$ cell counts remained unchanged (see online supplementary figure S3A-C). In a modified bleomycin model, in which we initiated pharmacological treatment after fibrosis had already been manifest, both inhibitors were effective in reversing established fibrosis to below pretreatment levels (figure $2 \mathrm{~A}-\mathrm{D}$ ).

\section{Inhibition of canonical Wnt signalling reduces AdTBRlact dermal fibrosis}

We have recently shown that TGF- $\beta$ stimulates canonical Wnt signalling and that the activation of canonical Wnt signalling contributes to the profibrotic effects of TGF- $\beta$. $^{3}$ Therefore, we examined the antifibrotic effects of PKF118-310 and ICG-001 in AdTBRIact challenged mice, as a model of TGF- $\beta$-driven fibrosis. Axin-2 mRNA levels were reduced in the skin from PKF118-310 and ICG-001 treated mice, again indicating effective inhibition of canonical Wnt signalling (data not shown). Both compounds were tolerated well with no changes in the clinical score (consistently 0 according to online supplementary table S1) and constant weight (see online supplementary figure S1C). Treatment with PKF118-310 or ICG-001 potently decreased dermal thickening, accumulation of collagen and differentiation of resting fibroblasts into myofibroblasts in AdTBRIact treated mice (figure $3 \mathrm{~A}-\mathrm{C}$ ), while the content of subcutaneous fat remained unchanged (see online supplementary figure S2B).

\section{DISCUSSION}

Due to the prominent role of canonical Wnt signalling in various types of cancers, tremendous efforts have been spent on developing novel therapeutics to target this pathway. This research, however, has long been unsuccessful, with the canonical Wnt pathway being considered 'undruggable'. ${ }^{18}$ It is only in recent years that novel strategies to inhibit canonical Wnt signalling up- or downstream of the central integrator $\beta$-catenin are emerging. ${ }^{19}$ In regard to SSc, upstream targeting of the canonical Wnt pathway may be limited by the diversity in

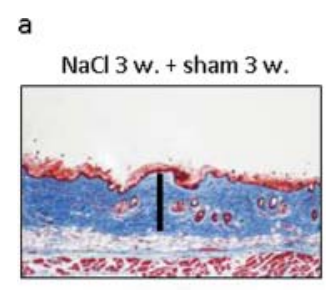

b

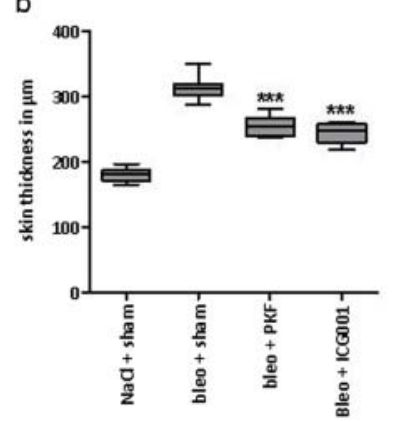

Bleo 3 w. + sham 3 w.

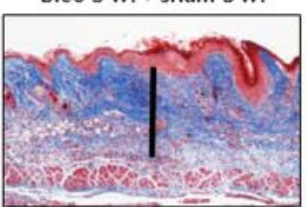

C

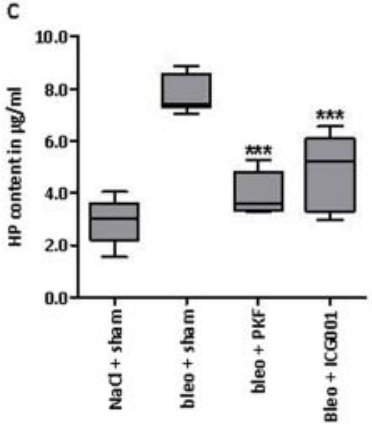

Bleo $3 w .+$ PKF $3 w$

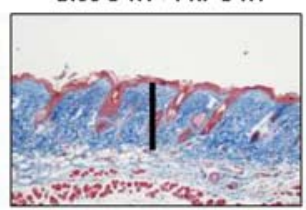

d

Figure 1 Inhibition of canonical Wnt signalling by PKF118-310 and ICG-001 prevents the development of bleomycin-induced skin fibrosis.(A) Representative trichrome stains with blue staining for collagens are shown at 100 -fold magnification. (B) Dermal thickening as determined by trichrome staining. (C) Hydroxyproline content. (D) $\alpha$ - smooth muscle actin (SMA) positive myofibroblasts per high power field. The $\mathrm{NaCl}$ and the bleomycin groups receiving sham treatment consisted of 10 animals each, the treatment groups with PKF118-310 and ICG-001 of eight animals each. Both treatment groups were tested for significant effects against the group with bleomycin challenge and sham treatment. ${ }^{*} 0.05>p>0.01$; ${ }^{* *} 0.01>p>0.001 ;{ }^{* * *} p<0.001$. 
a



b

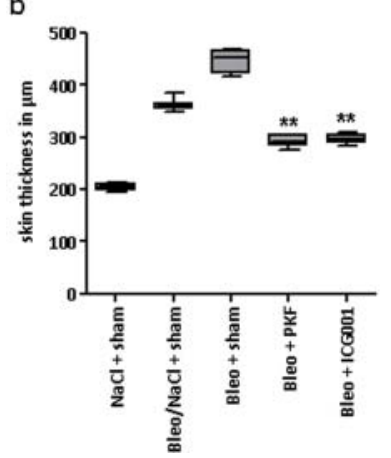

Bleo 3 w./NaCl $3 w$. + sham $3 w$.

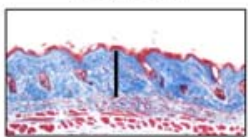

c
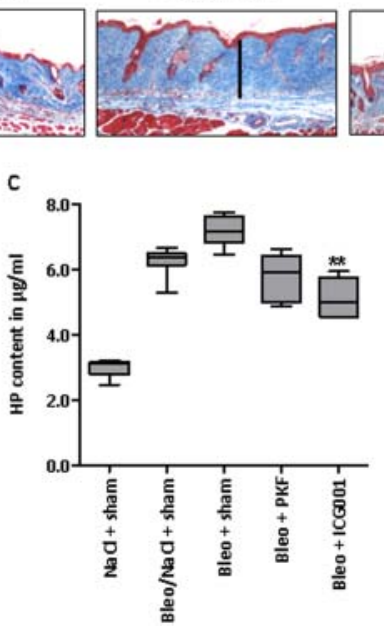

Bleo $6 \mathrm{w}$.

+ PKF $3 w$.

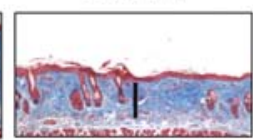

d

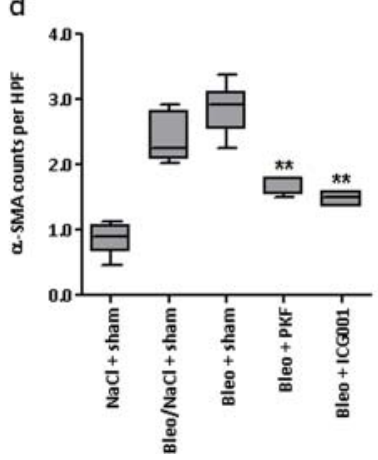

Figure 2 Inhibition of canonical Wnt signalling by PKF118-310 and ICG-001 induces the regression of established bleomycin-induced skin fibrosis. (A) Representative trichrome stains with blue staining for collagens are shown at 100 -fold magnification. (B) Dermal thickening as determined by trichrome staining. (C) Hydroxyproline content. (D) $\alpha$ - smooth muscle actin (SMA) positive myofibroblasts per high power field. The $\mathrm{NaCl}$ and the bleomycin groups receiving sham treatment consisted of eight animals each, the treatment groups with PKF118-310 and ICG-001 of five animals each. Both treatment groups were tested for significant effects against the group with 3 weeks of bleomycin/3 weeks with $\mathrm{NaCl}$ challenge and sham treatment. ${ }^{*} 0.05>p>0.01 ;{ }^{* *} 0.01>p>0.001 ;{ }^{* * *} p<0.001$.

Wnt receptors and ligands involved in the disease process: in SSc, Wnt-1 and Wnt-10b are overexpressed and activate canonical Wnt signalling. ${ }^{2}{ }^{3}$ In addition, decreased expression of endogenous inhibitors contributes to the aberrant activation of Wnt signalling in SSc. ${ }^{3}$ Thus, blockade of a single Wnt protein or receptor may be bypassed and therefore result in only limited antifibrotic effects. Although non-selective targeting of $\beta$-catenin may be effective in treating fibrosis, this approach is likely limited by toxicity: apart from its central role in canonical Wnt signalling, $\beta$-catenin is an important anchor protein in adherens junctions and therefore essential for cellular integrity. $^{2}$ While general targeting of $\beta$-catenin can interrupt intercellular contacts and disturb tissue organisation, selective modulation of the transcriptional $\beta$-catenin activity may offer both potent antifibrotic effects and high tolerability. In this context, we have already demonstrated that tankyrase inhibitors, which fine-tune the stability of the $\beta$-catenin destruction complex, are effective and well tolerated in experimental

a

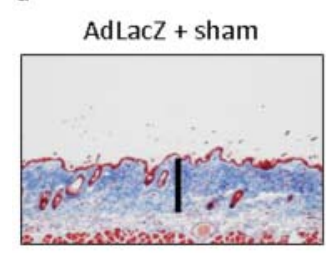

b

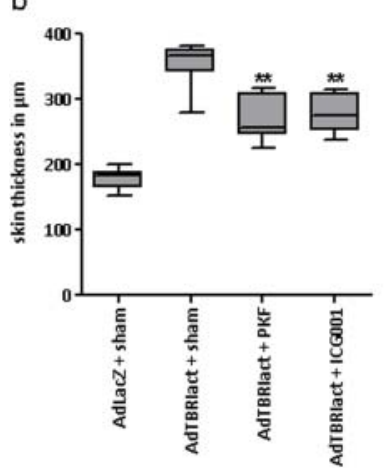

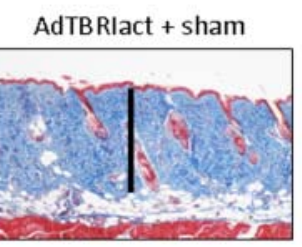

C

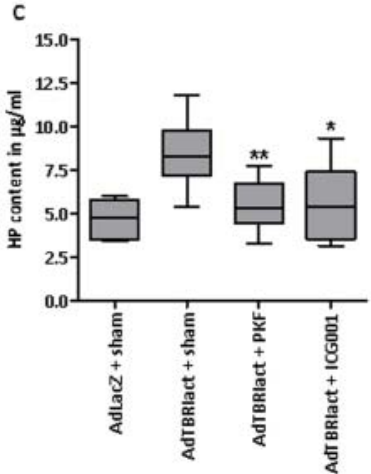

AdTBRlact + PKF

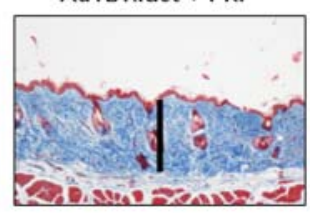

d

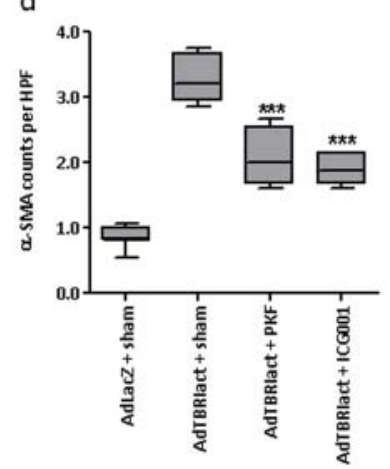

Figure 3 Inhibition of canonical Wnt signalling by PKF118-310 and ICG-001 inhibits skin fibrosis induced by attenuated adenoviruses overexpressing a constitutively active TGF- $\beta$ receptor I. (A) Representative trichrome stains with collagens in blue are shown at 100-fold magnification. (B) Skin thickening as determined by trichrome staining. (C) Hydroxyproline content. (D) $\alpha$ - smooth muscle actin (SMA) positive myofibroblasts per high power field. All groups consisted of eight samples each. Both treatment groups were tested for significant effects against the group with AdTBRlact challenge and sham treatment. ${ }^{*} 0.05>p>0.01 ;{ }^{* *} 0.01>p>0.001 ;{ }^{* * *} p<0.001$. 
fibrosis. $^{20}$ Targeting of Wnt-induced nuclear interactions of $\beta$-catenin with transcriptional regulators, as investigated in this study, may also overcome limitations of non-selective blockade of $\beta$-catenin. The complex formation of $\beta$-catenin with TCF transcription factors and the recruitment of co-activators (eg, $\mathrm{CBP}$ and p300) are essential for transcription of Wnt target genes. ${ }^{12}{ }^{18}$ Blockade of these processes may effectively inhibit profibrotic Wnt signalling, but would not interfere with other $\beta$-catenin functions and thus may be most promising in terms of efficacy and tolerability.

We therefore investigated the antifibrotic effects of targeting the $\beta$-catenin/TCF-interaction by PKF118-310 and the recruitment of co-activators by ICG-001. Our results provide the first evidence that these approaches have potent antifibrotic effects in different preclinical models of skin fibrosis. Both compounds effectively prevented and reversed inflammation-driven fibrosis and reduced TGF- $\beta$ dependent fibrosis, the latter resembling non-inflammatory stages of fibrosis. The efficacy in TGF- $\beta$-driven dermal fibrosis further highlights the close interaction of the TGF- $\beta$-pathway with canonical Wnt signalling and confirms that canonical Wnt signalling is a downstream mediator of TGF- $\beta$ in fibrosis. ${ }^{3}$ Our findings suggest that both strategies may be effective in various stages of skin fibrosis. Considering the profibrotic role of canonical Wnt signalling in various fibrotic diseases, our therapeutic strategies may also treat fibrosis of other organs. Indeed, first studies suggest that ICG-001 may also be effective in experimental models of renal and pulmonary fibrosis. ${ }^{15} 16$

Safety and tolerability are important issues when inhibiting canonical Wnt signalling. Tissues with high cell turnover, including the gastrointestinal tract or the hair follicles in the skin, may be affected by Wnt inhibition. ${ }^{12}$ In our study, however, we did not observe diarrhoea or weight loss as signs of gastrointestinal toxicity from impaired regeneration of the colonic epithelium. Moreover, gross macroscopic evaluation did not reveal alterations of the architecture of gastrointestinal crypts. Similarly, mice did not show hair loss, changes in the texture of the fur or macroscopic alterations of the hair bulb. Nevertheless, additional studies with more in-depths analyses of the different stem cell populations should further assess the safety of pharmacological Wnt inhibitors.

Taken together, we demonstrated that pharmacological inhibition of canonical Wnt signalling has potent antifibrotic effects in different experimental models of skin fibrosis. Wnt inhibitors that target $\beta$-catenin/TCF interaction or $\beta$-catenin co-factor recruitment may thus represent potential therapeutic approaches. Future studies, however, need to pay close attention to potential side effects of these therapies regarding the central role of canonical Wnt signalling in stem cell maintenance and tissue homeostasis.

Acknowledgements We thank Verena Wäsch and Madeleine Demleitner for excellent technical assistance.
Contributors Design of the study: $C B$, JD. Data acquisition: $C B, H R, H A, T M, A S$, $C D, K P Z, N Y L, A D, K G$. Manuscript preparation: CB, JD, JV, OD, GS.

Funding Grant support was provided by the Erlanger Leistungsbezogene Anschubfinanzierung und Nachwuchsföderung (ELAN), grants J29 and A40 of the Interdisciplinary Center of Clinical Research (IZKF) in Erlangen and grants DI 1537/1-1, DI 1537/2-1, DI 1537/4-1, AK 144/1-1, BE 5191/1-1 and SCHE 1583/7-1 from the Deutsche Forschungsgesellschaft. In addition, the study was supported by a doctoral scholarship from the Bayerische Forschungsstiftung (to TM and NYL) and the Career Support Award of Medicine of the Ernst Jung Foundation (to JD).

Competing interests None.

Provenance and peer review Not commissioned; externally peer reviewed.

\section{REFERENCES}

1. Wynn TA. Cellular and molecular mechanisms of fibrosis. J Pathol 2008;214:199-210.

2. Beyer C, Schramm A, Akhmetshina A, et al. beta-catenin is a central mediator of pro-fibrotic Wnt signaling in systemic sclerosis. Ann Rheum Dis 2012;11:761-7.

3. Akhmetshina A, Palumbo K, Dees $C$, et al. Activation of canonical Wnt signalling is required for TGF-beta-mediated fibrosis. Nat Commun 2012;3:735.

4. Bergmann C, Akhmetshina A, Dees C, et al. Inhibition of glycogen synthase kinase $3 \beta$ induces dermal fibrosis by activation of the canonical Wnt pathway. Ann Rheum Dis 2011;70:191-8.

5. Wei J, Melichian D, Komura K, et al. Canonical Wnt signaling induces skin fibrosis and subcutaneous lipoatrophy: a novel mouse model for scleroderma? Arthritis Rheum 2011;63:1707-17.

6. Colwell AS, Krummel TM, Longaker MT, et al. Wnt-4 expression is increased in fibroblasts after TGF-beta1 stimulation and during fetal and postnatal wound repair. Plast Reconstr Surg 2006;117:2297-301.

7. Konigshoff $\mathbf{M}$, Kramer M, Balsara N, et al. WNT1-inducible signaling protein-1 mediates pulmonary fibrosis in mice and is upregulated in humans with idiopathic pulmonary fibrosis. J Clin Invest 2009;119:772-87.

8. Surendran K, McCaul SP, Simon TC. A role for Wnt-4 in renal fibrosis. Am J Physio Renal Physiol 2002;282:F431-441.

9. Wei J, Fang F, Lam AP, et al. Wnt/ $\beta$-catenin signaling is hyperactivated in systemic sclerosis and induces Smad-dependent fibrotic responses in mesenchymal cells. Arthritis Rheum 2012;64:2734-45.

10. Gabrielli A, Avvedimento EV, Krieg T. Scleroderma. N Engl J Med 2009;360:1989-2003.

11. Varga J, Abraham D. Systemic sclerosis: a prototypic multisystem fibrotic disorder. J Clin Invest 2007;117:557-67.

12. van Amerongen R, Nusse R. Towards an integrated view of Wnt signaling in development. Development 2009;136:3205-14.

13. Beyer C, Schett G, Distler 0, et al. Animal models of systemic sclerosis: prospects and limitations. Arthritis Rheum 2010;62:2831-44.

14. Dees C, Akhmetshina A, Zerr P, et al. Platelet-derived serotonin links vascular disease and tissue fibrosis. J Exp Med 2011;208:961-72.

15. Hao S, He W, Li Y, et al. Targeted inhibition of $\beta$-catenin/CBP signaling ameliorates renal interstitial fibrosis. J Am Soc Nephrol 2011;22:1642-53.

16. Henderson WR Jr, Chi EY, Ye X, et al. Inhibition of Wnt/beta-catenin/CREB binding protein (CBP) signaling reverses pulmonary fibrosis. Proc Natl Acad Sci USA 2010;107:14309-14.

17. Hallett RM, Kondratyev MK, Giacomelli AO, et al. Small molecule antagonists of the $\mathrm{Wnt} /$ beta-catenin signaling pathway target breast tumor-initiating cells in a Her2/Neu mouse model of breast cancer. PLoS One 2012;7:e33976.

18. Verkaar F, Zaman GJ. New avenues to target Wnt/beta-catenin signaling. Drug Discov Today 2011;16:35-41.

19. Beyer C, Distler JH. Morphogen pathways in systemic sclerosis. Curr Rheumatol Rep 2013;15:299.

20. Distler A, Deloch L, Huang J, et al. Inactivation of tankyrases reduces experimental fibrosis by inhibiting canonical Wnt signalling. Ann Rheum Dis Published Online First 12 Nov 2012. doi:10.1136/annrheumdis-2012-202275 


\section{ARD Blockade of canonical Wnt signalling ameliorates experimental dermal fibrosis}

Christian Beyer, Helena Reichert, Hümeyra Akan, Tatjana Mallano,

Amelie Schramm, Clara Dees, Katrin Palumbo-Zerr, Neng Yu Lin, Alfiya Distler, Kolja Gelse, John Varga, Oliver Distler, Georg Schett and Jörg H W Distler

Ann Rheum Dis 2013 72: 1255-1258 originally published online April 17, 2013

doi: 10.1136/annrheumdis-2012-202544

Updated information and services can be found at:

http://ard.bmj.com/content/72/7/1255

These include:

Supplementary Supplementary material can be found at:

Material http://ard.bmj.com/content/suppl/2013/04/16/annrheumdis-2012-2025 44.DC1.html

References This article cites 19 articles, 6 of which you can access for free at: http://ard.bmj.com/content/72/7/1255\#BIBL

Email alerting Receive free email alerts when new articles cite this article. Sign up in the service box at the top right corner of the online article.

Topic Articles on similar topics can be found in the following collections Collections

\section{Notes}

To request permissions go to:

http://group.bmj.com/group/rights-licensing/permissions

To order reprints go to:

http://journals.bmj.com/cgi/reprintform

To subscribe to BMJ go to:

http://group.bmj.com/subscribe/ 